\title{
Research on Measured Course Accuracy Based on Relative Measure between Azimuth and Relative Bearing
}

\author{
Wang Hai-bo ${ }^{1, a}$, Xu kuo ${ }^{1, b}$, Zhou Ai-jun ${ }^{1, c}$, Hu Hong-can ${ }^{1, d}$ \\ ${ }^{1}$ Dept. of Navigation, Dalian Naval Academy, Dalian, 116018, China \\ a2046792022@qq.com, bhldwhb@foxmail.com, chldwhb@tom.com, xueshulenwen@163.com
}

Keywords: azimuth; relative bearing; relative measurement; theodolite; pitch angle and roll angle; measuring accuracy.

\begin{abstract}
In view of the course error caused by the performance descent of the ship navigation device, the principle and mathematical model of the ship course alignment by the application of the relative measure between the azimuth and the relative bearing is presented in the paper. By the application of the error treatment theory and MATLAB simulation, the accuracy influence of the course by the relative measure on the pitch angle, the roll angle, the angle of altitude and the angle of chord is analyzed. The conclusion is that the pitch angle and the roll angle are the major factors which will influence the measured course accuracy of the relative measurement. At the berthing state, if the measured course accuracy of the relative measure wants to meet the alignment accuracy, the best meteorological condition should be selected first, secondly the pitch angle and roll angle should be pay attention, and the measurement of the angle of chord and the angle of altitude should be adjusted.
\end{abstract}

\section{Introduction}

A strict zero correction must be carried on the installed navigation equipment during the shipbuilding. A zero mismatch of the navigation equipment will be caused by the hull deformation and parts ageing after launching for a period of time, and along with the time rising, a course error will be caused by the decreasing of gyro performance, so that the gyro navigation equipment can not meet the service requirement [1]. Therefore a measure method is needed to provide a high accuracy ship course which can measure course accuracy of gyro navigation equipment regularly, When the ship is berthing, a course measure method based on relative measure method between azimuth and relative bearing can meet the course correction. In view of above method, the course measure mathematical model is formulated in this paper, the measured accuracy of the course is analyzed, and the measure condition which can meet the course correction demand is presented.

\section{Principle and Mathematical Model of Relative Measure Between Azimuth and Relative Bearing}

The relative measure between bearing and relative bearing which can increase the measure accuracy of the azimuth and decrease the limit of the sea area detection and the route is an expanding use of Overlapping azimuth mark, The measure principle is shown in Fig.1.

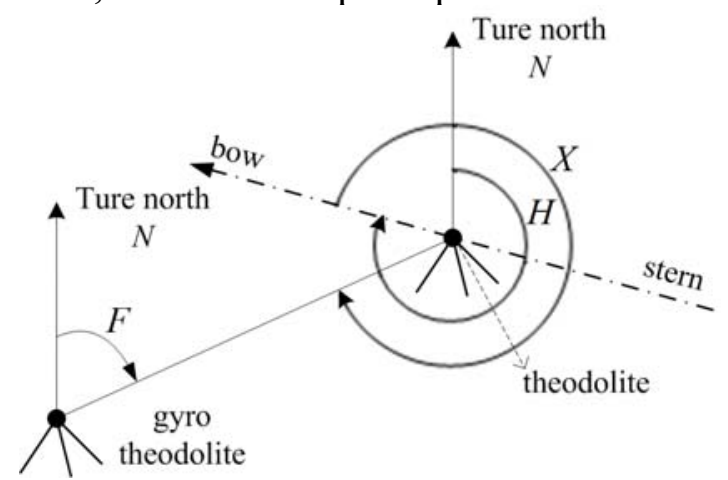

Fig.1 Principle of Relative Measure between Azimuth and Relative Bearing 
Set a gyro theodolite at a proper position on the shore, start gyro orientation after leveling the equipment, then get the true north azimuth. Set up the electronic theodolite on the fore and aft line of the ship deck, make the lens of the electronic theodolite aim at the heading line, set horizontal circle to zero. Rotate the telescope of the theodolite clockwise to relative measure with the gyro theodolite at the dock. At this very moment, the relative bearing measured by the electronic theodolite is $\mathrm{X}$, the azimuth measured by the gyro theodolite is F. Then the true course $\mathrm{H}$ of the ship can be obtained by equality (1).

$$
H=F-X+\gamma+\delta
$$

In equality (1), $\gamma$ is the meridian convergence angle of ship position, $\delta$ is the curvature correction of geodetic azimuth which is a relative value between ship position and gyro theodolite. In actual measurement, the distance between two theodolite is usually $10 \sim 15 \mathrm{~km}$, so $\delta$ and $\gamma$ can be ignored $^{[5]}$. Moreover, the pitch angle and the roll angle will lead to non-level of horizontal circle of telescope theodolite. In actual measurement, the treatment is to keep the deck plane parallel by regulating the horizontal circle. So the measured relative bearing and the angle of altitude are both relative deck coordinate system. So the relative bearing measured by telescope theodolite should be transformed from deck coordinate system to geographic coordinate system by utilizing the relative bearing error correction model in equality $(2)^{[6]}$.

$$
X_{t}=\arctan \left(\frac{\sin R \tan h_{b}+\cos R \sin X_{b}}{\sin R \sin P \sin X_{b}+\cos P \cos X_{b}-\cos R \sin P \tan h_{b}}\right)
$$

In equality (2), $X_{t}$ represents the true relative bearing of the geographic coordinate system, $X_{b}$ represents the relative bearing of the deck coordinate system, and $h_{b}$ represents the angle of altitude of the deck coordinate system, $P$ represents the pitch angle, and $R$ represents the roll angle. So it can be found that the true relative bearing is only influenced by the pitch angle $P$, the roll angle $R$, the relative bearing measured by the ship-board theodolite $X_{b}$ and the angle of altitude $h_{b}$.

So the navigation equipment, the gyro theodolite, the electronic theodolite enter the date at the same time, the true ship azimuth and the error $\Delta H$ of the measured navigation equipment can be obtained by calculation.

\section{Error Analysis of Relative Measure Between Azimuth and Relative Bearing}

On the basis of the system of the relative function of the error theory and the calculation processing method ${ }^{[7]}$, the measured azimuth accuracy can be obtained in equality (1).

$$
\sigma_{H}=\sqrt{\sigma_{F}^{2}+\sigma_{X_{t}}^{2}}
$$

In equality (3), $\sigma_{F}$ represents the measured azimuth accuracy; $\sigma_{X_{t}}$ represents the measured true relative bearing accuracy; the representation can be achieved as shown in equality (2):

$$
\sigma_{X_{t}}=\sqrt{\left(\frac{\partial X_{t}}{\partial P}\right)^{2} \sigma_{P}{ }^{2}+\left(\frac{\partial X_{t}}{\partial R}\right)^{2} \sigma_{R}{ }^{2}+\left(\frac{\partial X_{t}}{\partial h_{b}}\right)^{2}{\sigma_{h_{b}}}^{2}+\left(\frac{\partial X_{t}}{\partial X_{b}}\right)^{2}{\sigma_{X_{b}}}^{2}}
$$

In equality (4), $\sigma_{P}, \sigma_{R}$ represents the pitch angle accuracy and the roll angle accuracy, $\sigma_{h_{b}}$, $\sigma_{X_{b}}$ represents the accuracy of the azimuth and the angle of altitude measured by theodolite, the measured accuracy of electronic theodolite is at arc-second order, The best outcome is $1 " \sim 2$ ".$\frac{\partial X_{t}}{\partial P}$ 、

$$
\begin{gathered}
\frac{\partial X_{t}}{\partial R} 、 \frac{\partial X_{t}}{\partial h_{b}} 、 \frac{\partial X_{t}}{\partial X_{b}} \text { are the error transmission coefficient of the true relative bearing. } \\
\frac{\partial X_{t}}{\partial P}=-\frac{\tan X_{t}\left(\sin R \cos P \sin X_{b}-\sin P \cos X_{b}-\cos R \cos P \tan h_{b}\right)}{\sec ^{2} X_{t}\left(\sin R \sin P \sin X_{b}+\cos P \cos X_{b}-\cos R \sin P \tan h_{b}\right)}
\end{gathered}
$$




$$
\begin{aligned}
\frac{\partial X_{t}}{\partial R} & =\frac{\cos R \tan h_{b}-\tan X_{t}\left(\cos R \sin P \sin X_{b}+\sin R \sin P \tan h_{b}\right)-\sin R \sin X_{b}}{\sec ^{2} X_{t}\left(\sin R \sin P \sin X_{b}+\cos P \cos X_{b}-\cos R \sin P \tan h_{b}\right)} \\
\frac{\partial X_{t}}{\partial h_{b}} & =\frac{\tan X_{t} \cos R \sin P \sec ^{2} h_{b}+\sin R \sec ^{2} h_{b}}{\sec ^{2} X_{t}\left(\sin R \sin P \sin X_{b}+\cos P \cos X_{b}-\cos R \sin P \tan h_{b}\right)} \\
\frac{\partial X_{t}}{\partial X_{b}} & =-\frac{\tan X_{t}\left(\sin R \sin P \cos X_{b}-\cos P \sin X_{b}\right)-\cos R \cos X_{b}}{\sec ^{2} X_{t}\left(\sin R \sin P \sin X_{b}+\cos P \cos X_{b}-\cos R \sin P \tan h_{b}\right)}
\end{aligned}
$$

It can be achieved through formula derivation and analysis that the factors which influence the course measure accuracy are not only the pitch angle accuracy, the roll angle, the angle measurement accuracy of electronic theodolite, but also the azimuth measure accuracy. But considering the accuracy of the gyro theodolite is high in general, so the influence of the azimuth accuracy can be ignored. So the error of the course $\Delta H$ can be considered similarly equal with the error of the relative bearing $\Delta X$.

When the ship course is measured at berthing state, the ship sway extent is low, and the error of the relative measure is less. Compared with the navigation state, if the weather condition is good, it will be more practical to measure the course to adopt the relative measure method. So the effect of the pitch angle, the roll angle, the relative bearing and the angle of altitude on the relative bearing measure accuracy at the berthing state is analyzed in the following paper.

The value in the geographic coordinate system is replaced by the relative bearing measured directly by the electronic theodolite at berthing state, is regarded as a truth value, and the error of the relative bearing can be obtained as shown in equality (9).

$$
\Delta X=X_{b}-X_{t}=X_{b}-\arctan \left(\frac{\sin R \tan h_{b}+\cos R \sin X_{b}}{\sin R \sin P \sin X_{b}+\cos P \cos X_{b}-\cos R \sin P \tan h_{b}}\right)
$$

It can be known from equality (9) that the factors influencing the error of the relative bearing are the pitch angle, the roll angle, the relative bearing and the angle of altitude. So how to choose a proper condition to ensure the whole measure accuracy is difficult and important in the relative measure method, the influence of these factors on the error of the relative bearing is analyzed in the following paper.

\section{Simulation Analysis}

Man-machine interaction interface of high level visualization is designed with the use of GUI environment supplied by MATLAB in the simulation. The measured value is inputted manually, the azimuth is measured in the relative measure method at berthing state in the simulation, the error of the measured relative bearing caused by the pitch angle, the roll angle, the measured relative bearing and the angle of altitude is simulated one by one, he program design flowchart is shown in Fig. 2.

Simulation Analysis of Influence of Pitch Angle and Roll Angle on Error of Relative Bearing. Simulation condition: the amplitude of the pitch angle and the roll angle is respectively $-0.3^{\circ} \sim 0.3^{\circ}$, $-0.5^{\circ} \sim 0.5^{\circ}$, the angle of altitude is $1^{\circ}$, the relative bearing is $-150^{\circ}$. It is shown in Fig. 3 that the error of the relative bearing increases followed the increase of the pitch angle and the roll angle, the change of the pitch angle and the roll angle has a strong influence on the measure accuracy. Moreover, a large number simulations shows that if the pitch angle and the roll angle increase, the error will increase followed it, where, the influence of the roll angle is larger than the pitch angle.

Simulation Analysis of Influence of Measured Relative Bearing on Error of Relative Bearing. Simulation condition: the amplitude of the pitch angle and the roll angle are both $0.3^{\circ}$, the angle of altitude is respectively $0^{\circ}$ and $1^{\circ}$, the relative bearing is $-180^{\circ} \sim 180^{\circ}$. It is shown in Fig. 4 (when the angle of altitude is $0^{\circ}$ ) that the measured relative bearing has an influence on the error of the relative bearing, and the relative bearing measured with the gyro theodolite when the ship is abeam positioned has a max influence on the error of the relative bearing. It is shown Fig.5 that when the angle of altitude changes, the measured relative bearing which is corresponding with the peak 
error value of relative bearing will change thereupon, so the operation should be adjusted on the base of the corresponding accuracy demand and the specific situation.

Simulation Analysis of Influence of Measured Angle of Altitude on Error of Relative Bearing. Simulation condition: the amplitude of the pitch angle and the roll angle are both $0.1^{\circ}$, the angle of altitude is $-3^{\circ} \sim 3^{\circ}$, the relative bearing is $-150^{\circ}$. It is shown in Fig. 6 that the error of the relative bearing increases when the angle of altitude changes from $-3^{\circ}$ to $3^{\circ}$, the max error is $26^{\prime \prime}$. Furthermore, the larger the measured amplitude angle of altitude is, the larger the measured error is.

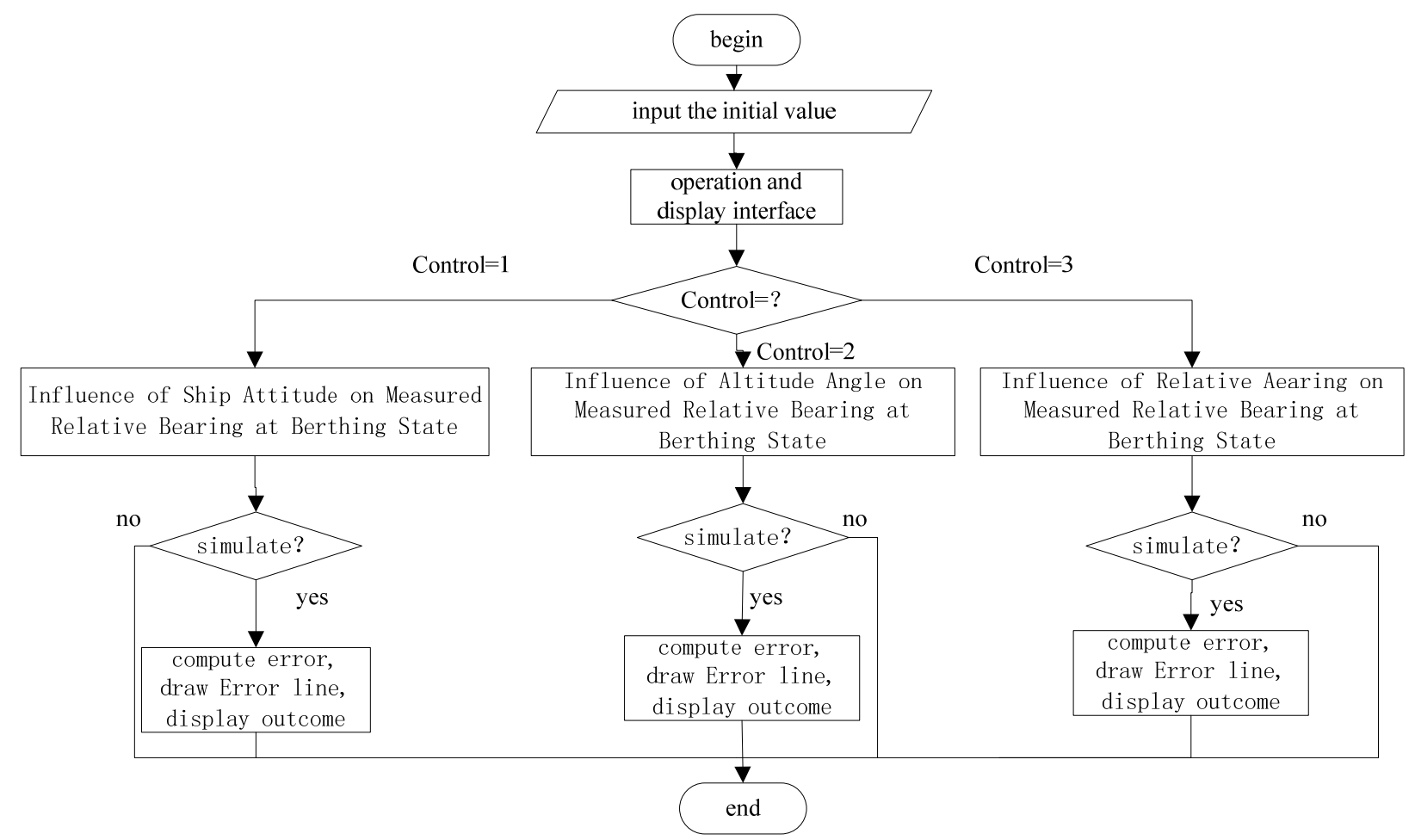

Fig.2 Program Design Flowchart

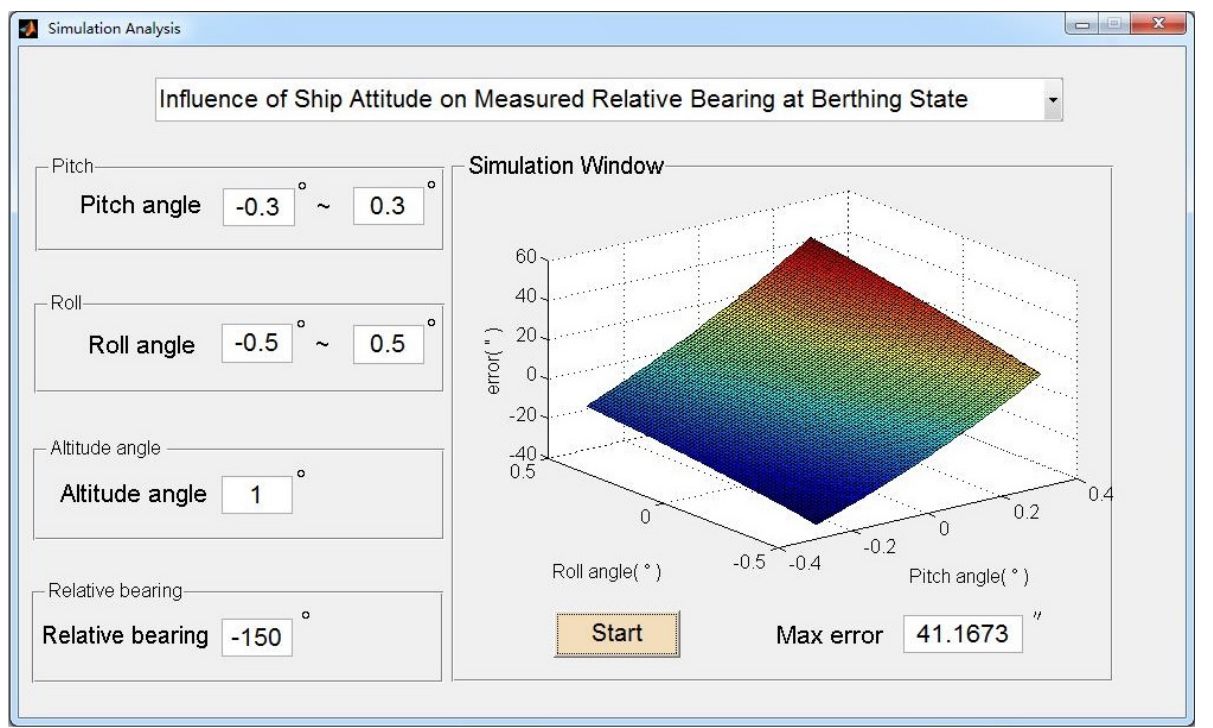

Fig.3 Influence of Ship Attitude on Measured Relative Bearing at Berthing State 


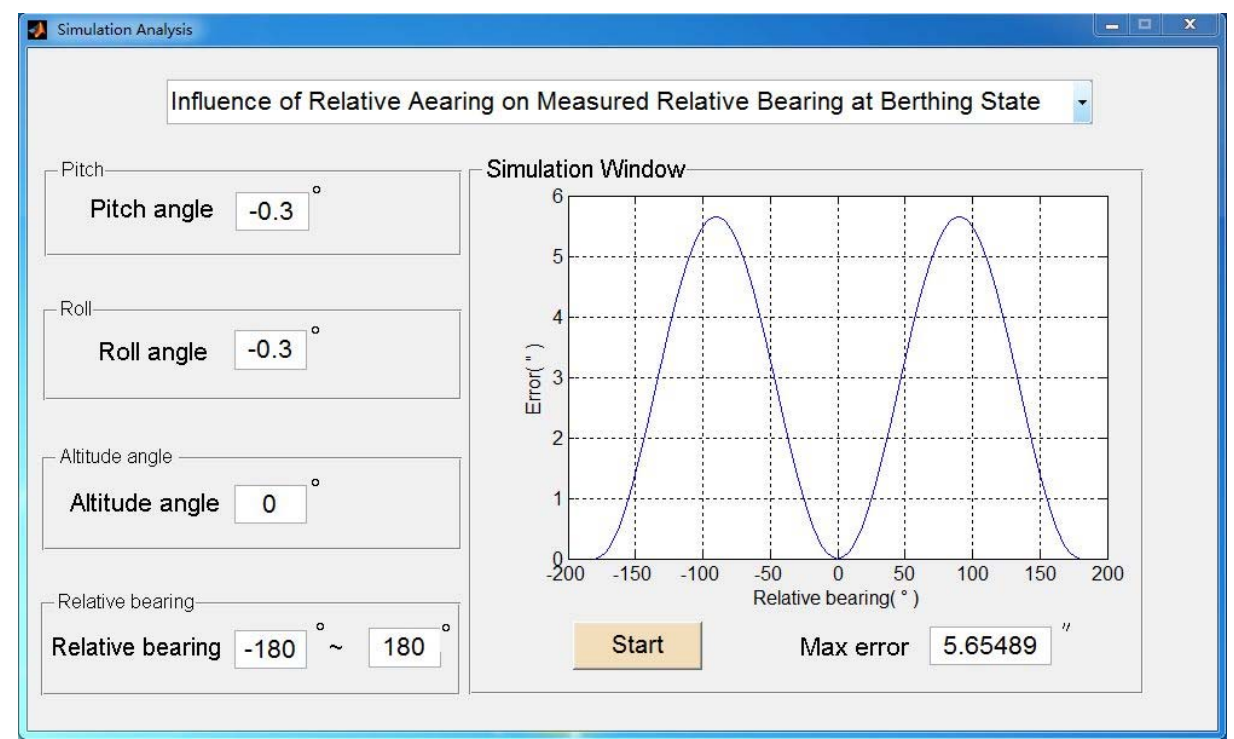

Fig.4 Influence of Measured Relative Bearing on Error of Measured Relative Bearing at Berthing State(a)

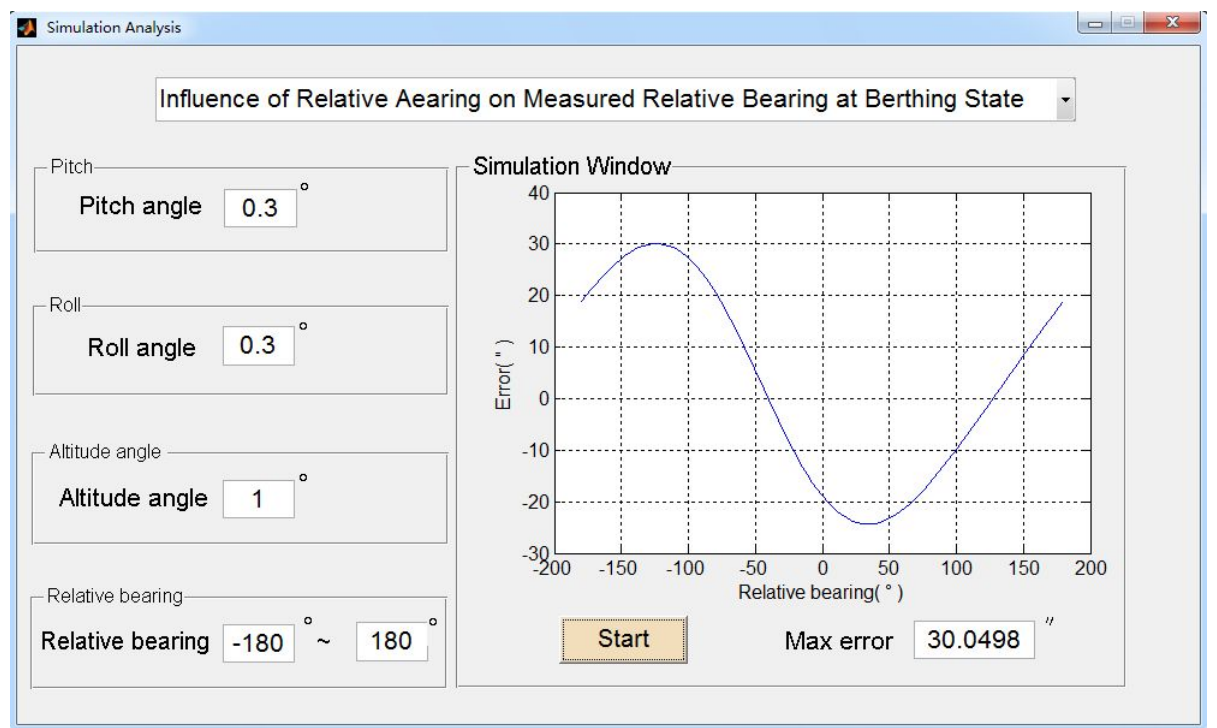

Fig.5 Influence of Measured Relative Bearing on Error of Measured Relative Bearing at Berthing State(b)

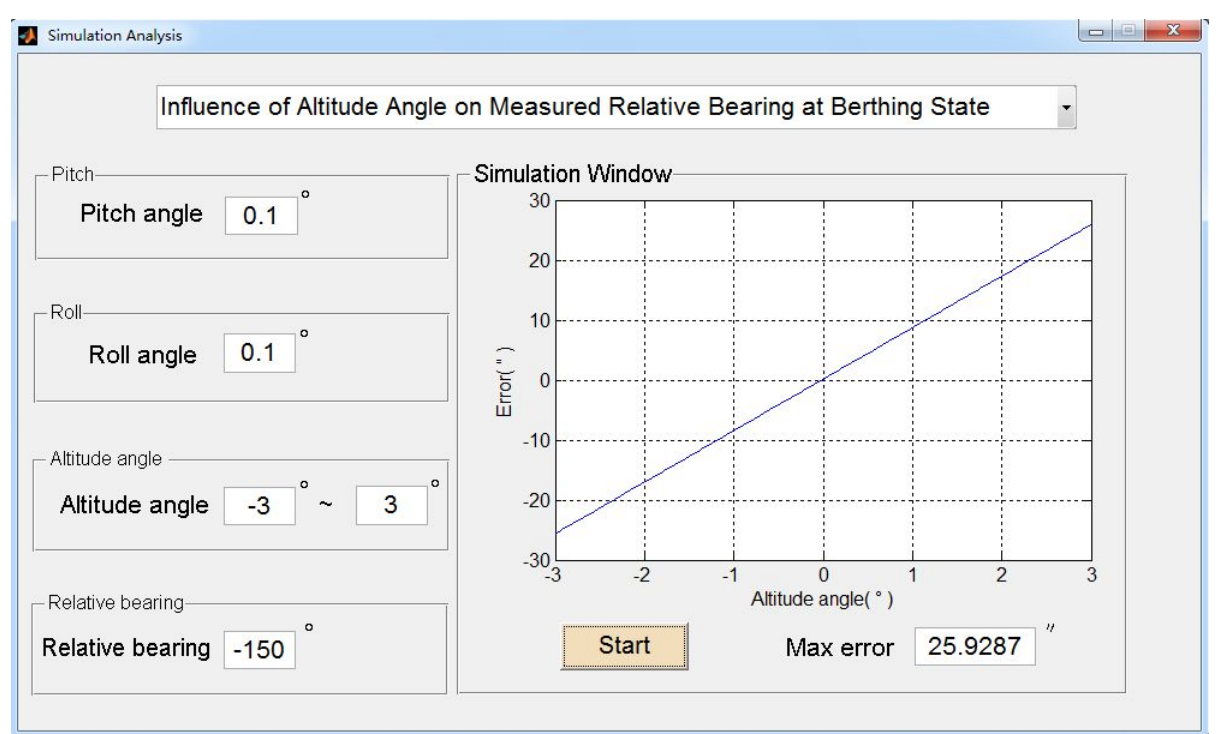

Fig.6 Influence of Measured Angle of Altitude on Error of Measured Relative Bearing at Berthing State

It can be concluded from above analysis that the course accuracy of the relative measure is influenced by the pitch angle, the roll angle, the measured relative bearing and the measured angle of altitude. It is shown in a large number of simulation that the pitch angle and the roll angle are the 
principal factors, and the influence of the roll angle is larger than the pitch angle. To acquire the optimal result, the corresponding factors such as the weather situation and the hydrology condition should be taken notice in specific operation.

\section{Conclusion}

We come to the conclusion from above theoretical and simulated analysis:

1)The course accuracy of the relative measure is influenced by the pitch angle, the roll angle, the measured relative bearing and the measured angle of altitude, and these influences of different factors are not same, the major influencing factors are the pitch angle and the roll angle.

2)The relative measure is suitable for course correction when the ship is at berthing state, and the pitch angle and the roll angle is demanded to be small. The measure should be operated after the measured angle of altitude and the relative bearing are adjusted properly when the measured course accuracy can not meet the correction accuracy demand.

3)When the amplitude of the pitch angle and the roll angle are a bit large, the measured accuracy of the course of the relative measure can not meet the demand of correction accuracy, and the error will be large, then the measure operation should be stopped or be carried out at another wharf.

4)To ensure the angle accuracy of the theodolite, besides the factors mentioned above for the relative measure, the measure operation should be carried out in a good meteorological condition.

\section{References}

[1] X.T. Wei, X.Q. Kong, Y. Li. Gyrotheodolite Calibration Medthod Based on Optoelectronic Measuring. OPTICS \& OPTOELECTRONIC TECH NOLOGY, 2007,5(1):50-52.

[2] J.J. Chen, J.H. Liu, Q.L. Wang, Z.L. Yuan. Analysis on Orientation Accuracy of Gyrotheodolite. GEOMATICS \& SPATIAL INFORMATION TECHNOLOGY ,2008,31(5):49-50.

[3] G.J. Hu, About the I Angle Error Correction of Electronic Theodolite. Metrology \& Measurement Technique,2013,40(4):10-11.

[4] J.M. Guo, X.Y. Kong, Z.Q. Liu. Base of geodetic survey. WuChang: Wuhan University press, 2008.

[5] Z.Y. Zhang, G.F. Luo. Coordinate transformation of warship pose and analysis of stabilization compensation. Ship Science and Technology, 2009, 1(4):34-40.

[6] Z.L. Ding. Error theory and data processing. Harbin: Harbin Institute of Technology press,2002.

[7] Z.Y. Zhang. MATLAB tutorial (R2014a version). Beijing: Beijing university of aeronautics and astronautics press, 2007. 